\title{
Two-component jets from 3-dimensional magnetohydrodynamic jet simula- tions of disk winds at sub-parsec scales
}

\author{
Jan Staff ${ }^{1,}$ a , Nico Koning ${ }^{2}$, Rachid Ouyed ${ }^{2}$, and Ralph Pudritz ${ }^{3,4}$ \\ ${ }^{1}$ Department of Physics and Astronomy, Macquarie University NSW 2109, Australia \\ ${ }^{2}$ Department of Physics and Astronomy, University of Calgary, 2500 University Drive NW, Calgary, Alberta, T2N 1N4 Canada \\ ${ }^{3}$ Department of Physics and Astronomy, McMaster University, Hamilton ON L8S 4M1, Canada \\ ${ }^{4}$ Origins Institute, ABB 241, McMaster University, Hamilton ON L8S 4M1, Canada
}

\begin{abstract}
We explore the effect of large scale magnetic field on the formation of two-component jets in magnetohydrodynamic disk winds simulations. Our simulations show a one-component and two-component jets develop depending on the magnetic field distribution along the surface of the accretion disk. Magnetic field configurations with the least steep gradient along the disk lead to a well defined two-component jet with the self-similar (Blandford-Payne) configuration separating the two regimes. Our results have direct implications to jets models of AGN and GRBs if indeed two-component jets emanate directly from the accretion disk. Our findings imply that a three-component jets may exist in AGN jets if one takes into account a Blandford-Znajek component in the innermost, relativistic, regions.
\end{abstract}

\section{Introduction}

Astrophysical jets are commonly observed in many astrophysical settings involving a central object surrounded by an accretion disk (protostars, active galactic nuclei (AGNs), X-ray binaries, etc). These jets all have in common the accretion disk threaded by large-scale poloidal field lines. These jets are believed to result from the centrifugal acceleration of disc material by the magnetic field ([1]) which also acts as the collimating agent. The role of the magnetic field has been demonstrated in numerical simulations (e.g. [8]) where it was also shown that a universal scaling from YSO jets to AGN jets is feasible. Others (e.g. [11]) also find that the intrinsic jet acceleration mechanism is similar in both the AGN and the YSO systems and that jets in AGN, for example, can be looked at as scaled up, relativistic versions of the jets in YSOs. The simulations discussed here can naturally be scaled by assuming different central objects. In the context of AGNs, our results should be interpreted in the context of slowly rotating (to non-rotating) black holes systems. We highlight and focus on two-component jet solutions found in our simulations, which may be needed to reconcile the unification scheme of BL Lacs and FR I radio galaxies.

\section{Methods}

In all simulations the initial density profile is that of a hydro-statically stable accretion disk corona, with $\rho \propto$ $r^{-3 / 2}$. The disk follows a similar density profile, but it is

a. e-mail: jan.staff@mq.edu.au a factor 100 larger which is imposed by pressure balance between the disk surface and the overlaying corona. An initially current-free $(\nabla \times \mathbf{B}=0)$ magnetic field is set up in the corona that extends into the disk [5]. On the surface of the disk, the poloidal magnetic field strength falls off as a power law with disk radius : $B_{p} \propto r^{\mu-1}$. In order to investigate the role of the initial magnetic geometry in launching and collimating disk winds, we set up four different cases, $\mu=-0.01$ ([8], hereafter OP), the self-similar configuration of Blandford-Payne ([1], hereafter BP) with $\mu=-0.25$, and an $\mu=-0.5$ configuration ([10], hereafter $\mathrm{PP})$. In addition we investigated the the $\mu=-0.12$ which is intermediate between the OP and the BP cases. These range from conditions of most gradually falling and initially somewhat collimated fields to fields which fall off steeply with disk radius and that have much more open magnetic geometries.

We use a Cartesian grid. The initial conditions are a $10^{8} M_{\odot}$ non-spinning black hole as a point mass at the center of a Keplerian disk that provide the boundary conditions for the simulation. The inner edge of the accretion disk surrounding this black hole is at $10 r_{\mathrm{g}}$ with $r_{\mathrm{g}}=$ $2 G M / c^{2}$ being the Schwartzschild radius, $G$ is the gravitational constant, $M$ is the mass of the black hole and $c$ is the speed of light. The velocity at the inner edge of the disk in Keplerian rotation at this distance is $6.7 \times 10^{4} \mathrm{~km} / \mathrm{s}$ much below the speed of light. The disk has an outer radius of $800 r_{\mathrm{g}}$ far exceeding the size of the disk participating in the outflow.

The grid size is 1536 zones in the $x_{1}$ direction, corresponding to $3 \times 10^{4} R_{\mathrm{g}}(\sim 0.29 \mathrm{pc})$, and 500 zones in each of the $x_{2}$ and $x_{3}$ directions corresponding to $\pm 9000 R_{\mathrm{g}}(\sim 0.09$ 
pc). This particular box size was carefully chosen to contain the jet within the simulated domain. Incorrect results may be obtained if much mass leaves the grid. Once the front of the jet reaches the outer edge of the grid, we stop the simulation. We use ZeusMP to run our simulations ${ }^{1}$. We refer to [12] for more details on the setup.

\section{Results}

In Figure 1 we show the four jets that we have simulated just before they leave the box. The OP jet evolves the fastest, while the jet evolves slower with more negative $\mu$. All jets have a strong bow shock in the front of the jet. Despite the OP jet propagating forward the fastest, because of the different magnetic field structures a more negative $\mu$ leads to a higher Mach number at the bow shock in front of the jet. Therefore, the bow shock is bent backwards more sharply for the more negative $\mu$, explaining why the cocoon remains closer to the jet axis. This cocoon is only visible in the front part of the jet for OP and $\mu=-0.12$, as it has been pushed off the grid farther back. For the BP and PP simulations, it remains on the grid for the entire length of the jet.

In all simulations, the jet is rotating (see Fig. 2). The part of the jet close to the axis in the OP, $\mu=-0.12$ and BP simulations rotates with a Keplerian profile similar to the disk. The part of the PP jet closest to the disk also rotates with a Keplerian profile. Further from the source in the PP jet, as well as at large distances from the source in the jet in BP, $\mu=-0.12$, and OP jets, a kink mode develops forming a spiral-like structure that is able to wash out the Keplerian rotation profile. While the kink mode appears to grow farther from the source, it does not appear to grow out of control destroying the jet. Farther from the axis (in OP, $\mu=-0.12$, and $\mathrm{BP}$ ) the jet rotates faster than what should be expected if the whole jet was rotating as one Keplerian profile, showing that these jets have two components : an inner and an outer jet component.

In Fig. 1 we see that in all cases, the magnetic field wraps up tightly around the thin inner jet. We can see also from Fig. 1 that there is a helical field associated with the outer jets in the OP, $\mu=-0.12$, and BP simulations. This, however, appears to be more loosely twisted than around the inner jet. This helical field associated with the outer jet is clearly separated from the inner jet, another indication that the two components are dynamically and physically separated. There is no helical field twisting up outside of the thin jet in the PP simulation, as expected since there is no-outer-jet in this case. The outer jet component in the BP configuration is quite weak, and it terminates soon upon interacting with the cocoon of material pushed aside by the jet. We find no outer jet in the PP simulation.

From a $10^{8} M_{\odot}$ black hole, we find jet rotation velocities up to $55 \times 10^{3} \mathrm{~km} / \mathrm{s}$ for the inner OP jet, and up to $40 \times 10^{3} \mathrm{~km} / \mathrm{s}$ for the inner BP jet. The outer jet is clearly visible in the plot of the rotational velocity in Fig. 2, with

1. ZeusMP is a non-relativistic code, but by setting the inner edge of the disk at $10 r_{\mathrm{g}}$ the resulting velocities are sufficiently small that relativistic effects may be ignored. rotational velocity up to $20 \times 10^{3} \mathrm{~km} / \mathrm{s}$ in the OP jet. The more negative $\mu$ simulations have slower rotating outer jets, in the BP simulation the outer jet rotates with only a few thousand $\mathrm{km} / \mathrm{s}$.

In Fig. 3 we plot the specific angular momentum, which is conserved along a field line, given by $l=r v_{\phi}-$ $r B_{\phi} / \kappa$, where $r$ is the distance from the axis, $v_{\phi}$ is the toroidal velocity (in cylindrical coordinates), $B_{\phi}$ is the toroidal magnetic field, $\kappa=\frac{\rho v_{\mathrm{p}}}{B_{\mathrm{p}}}$ is the conserved mass loading along a given field line, $\rho$ is the mass density, $v_{\mathrm{p}}$ is the poloidal velocity, and $B_{\mathrm{p}}$ is the poloidal magnetic field strength [9] for all four jets. Note in particular that in the region of high toroidal velocity (Fig. 2), there is an increased negative specific angular momentum. This is because more of the angular momentum is being carried by the twisted toroidal field than the rotating fluid. The BP jet only shows a weak enhancement in $l$ associated with its outer jet, consistent with that outer jet being weak. The PP jet does not show any such enhancement, showing that the $\frac{r B_{\phi}}{\kappa}$ term does not dominate. It appears and this is somewhat expected that the self-similar configuration of BP separates the one-component from the two-component regimes.

The jets have an onion like velocity structure [12], with the fastest velocities found in the inner jet close to the axis, while the outer jet is much slower. This is reminiscent of the fast spine, slower sheath structure reported in several jets, for instance M87 (e.g. [6]).

The geometry's role becomes apparent when looking at the momentum vectors in Fig. 4, taken in a slice through the middle of the grid. In all cases, some of the wind from the disk is collimated into a thin (inner) jet. But, with the more open field configurations (where the field lines on the disk have a larger angle from the jet axis), some of the wind flows out at very large angles with the jet axis. This is especially noticeable in the PP simulation, where far from the disk axis, but close to the disk boundary, the momentum vectors are aligned almost perpendicular to the jet axis. As a consequence, a cavity is being cleared out very near the disk boundary. In the less open field configurations, the vectors have a smaller angle with the axis, and the material flowing out in that direction ends up being collimated into the outer jet. There is, however, a bit of the same cavity being cleared out also in these cases, but it is narrower. This cavity acts as a separation between the inner and outer jet.

The inner jet in the OP and $\mu=-0.12$ simulations, appears to open up with a more or less constant opening angle. We find that the opening angle in the OP jet is $7.0^{\circ}$, while in the $\mu=-0.12$ jet it is $5.6^{\circ}$. The inner BP jet and the PP jet appears to re-collimate further out in the jet.

\section{Summary}

We have presented the results of four 3D MHD simulations of winds from accretion disks around non-spinning black holes. Initially all models are set up the same way, with the only difference between the simulations being the opening angle of the magnetic field on the disk, or in other 


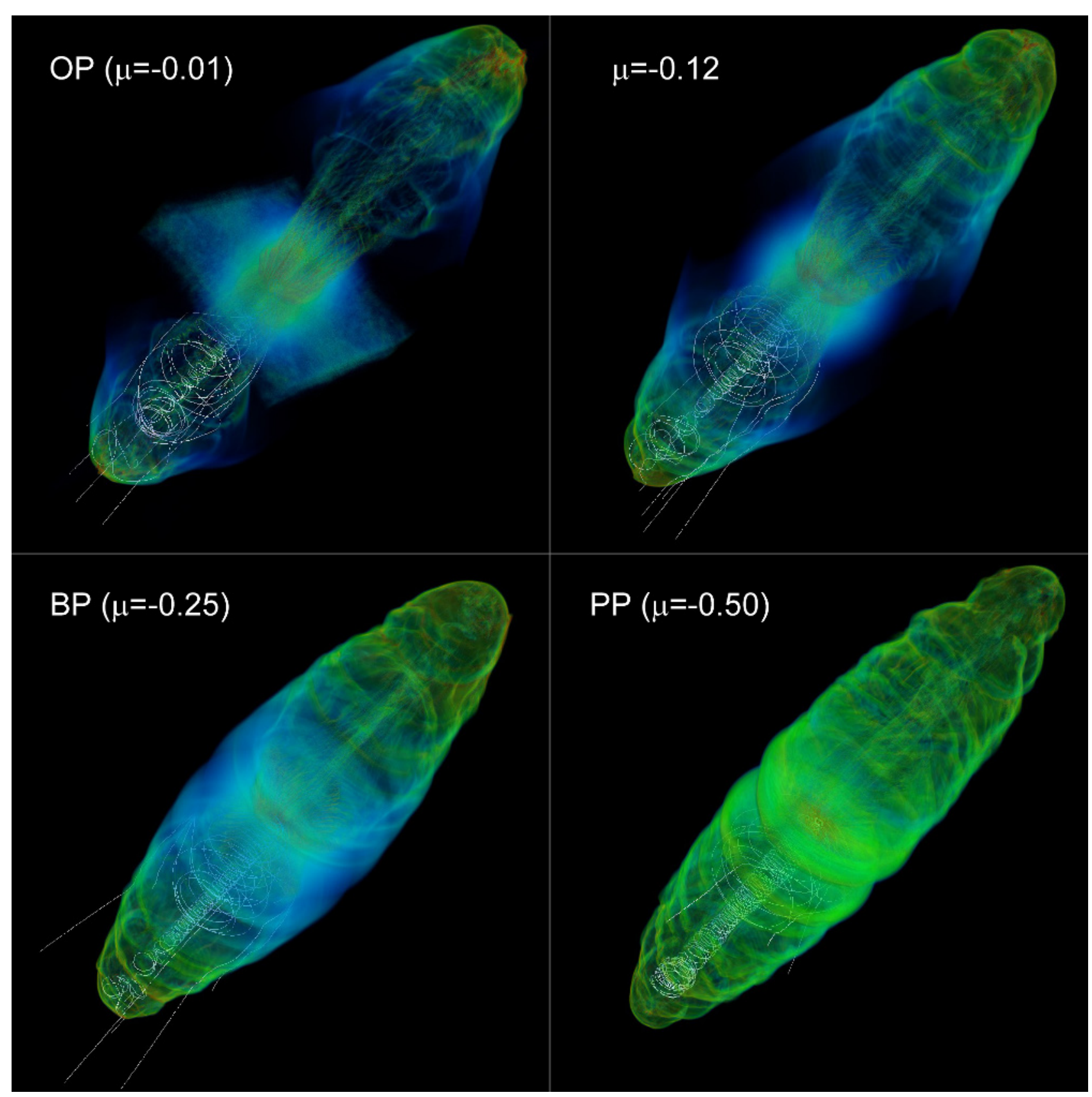

Figure 1. The density and magnetic field structure of the four jets resulting from the four increasingly open initial magnetic geometries for the disk/halo magnetic field configurations that we have simulated. The magnetic field is drawn on only one side to better illustrate the density structure. upper right : OP configuration, upper left : $\mu=-0.12$ configuration, lower left : BP configuration, lower right : $\mathrm{PP}$ configuration. Notice the clear inner and outer jet structure in the OP and $\mu=-0.12$ jets, and the weak outer jet structure in the BP jet. The BP and PP jets are surrounded by a cocoon of material pushed aside by the jet. This cocoon can be seen near the front of the OP and $\mu=-0.12$ jets, further back it has been pushed off the side of the simulation box.

words how fast the poloidal magnetic field on the disk drops off with radius. We find that a two-component jet develops in all cases except the simulation where the magnetic field drops off the fastest. The outer jet component is dynamically and physically separated from the inner jet.

The two-component jets we find from our simulations could be of relevance to AGN jets where it has been suggested that a two-component jet model may be needed to reconcile the viability of the unification scheme of BL Lacs and FR I radio galaxies (e.g. [4]). In the suggested two-component structure of AGN jets, a fast spine is surrounded by a slow (but still relativistic) layer not unlike what we are finding, assuming the scaling in velocities are justifiable. However, while proposed models seem to combine a Blandford-Znajek ([3]) and BP processes, we instead suggest that is not necessarily the case since a twocomponent jet can naturally arise from the disk itself. Our results might also be of relevance to GRBs which seem to require a two-component explosion involving two-jets (e.g. [2]). Finally, we note that a 3-component jet structure 


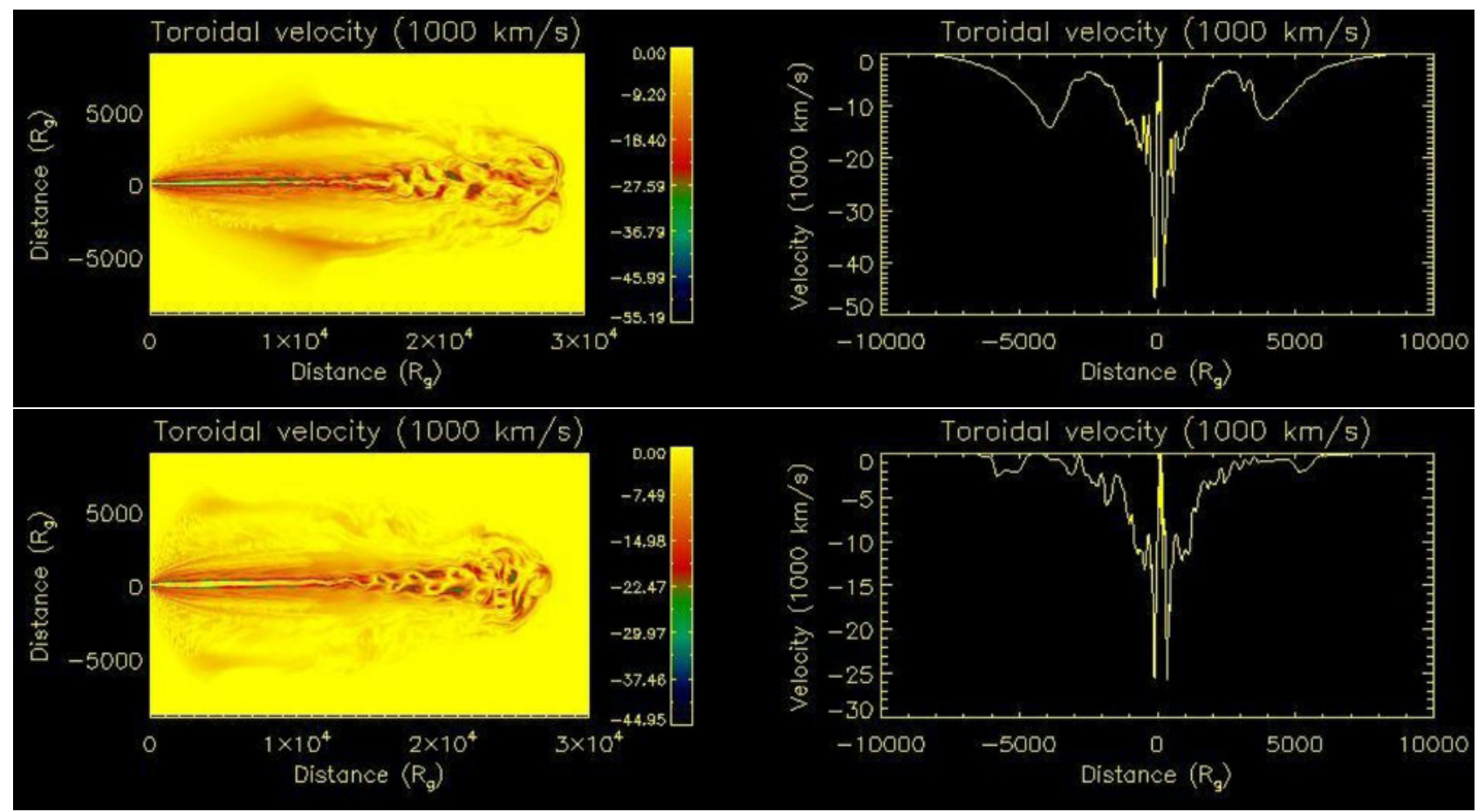

Figure 2. Contour plot of the velocity component in a slice through the middle of the grid for the OP simulation (top left) and BP simulation (bottom left). The right shows a plot taken across the jet at $10^{4} R_{\mathrm{g}}$ from the disk. The black hole mass is $10^{8} M_{\odot}$. We only plot the OP and BP simulations to save space, as these illustrate the general trend with lower toroidal velocity in the outer jet for more negative $\mu$.

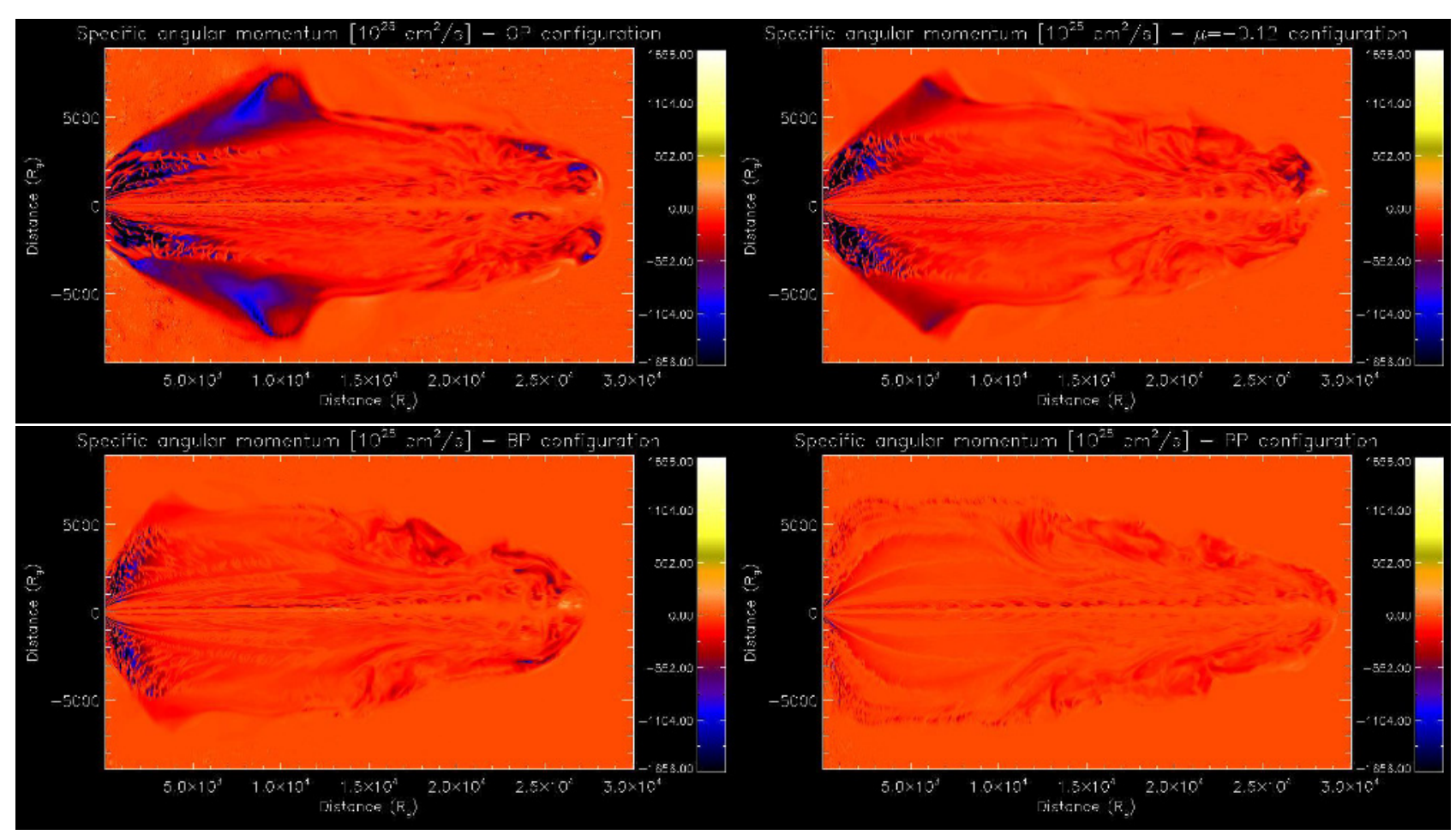

Figure 3. Specific angular momentum in a slice through the middle of the grid. The outer jet is clearly associated with a very strong specific angular momentum, in the OP simulation it is more than $10^{28} \mathrm{~cm}^{2} / \mathrm{s}$ (blue color), compared to the $\sim 10^{27} \mathrm{~cm}^{/} \mathrm{s}$ (red color) in the rest of the jet. The PP jet, without an outer jet, has low specific angular momentum $\sim 10^{27} \mathrm{~cm}^{2} / \mathrm{s}$ throughout the whole jet. 

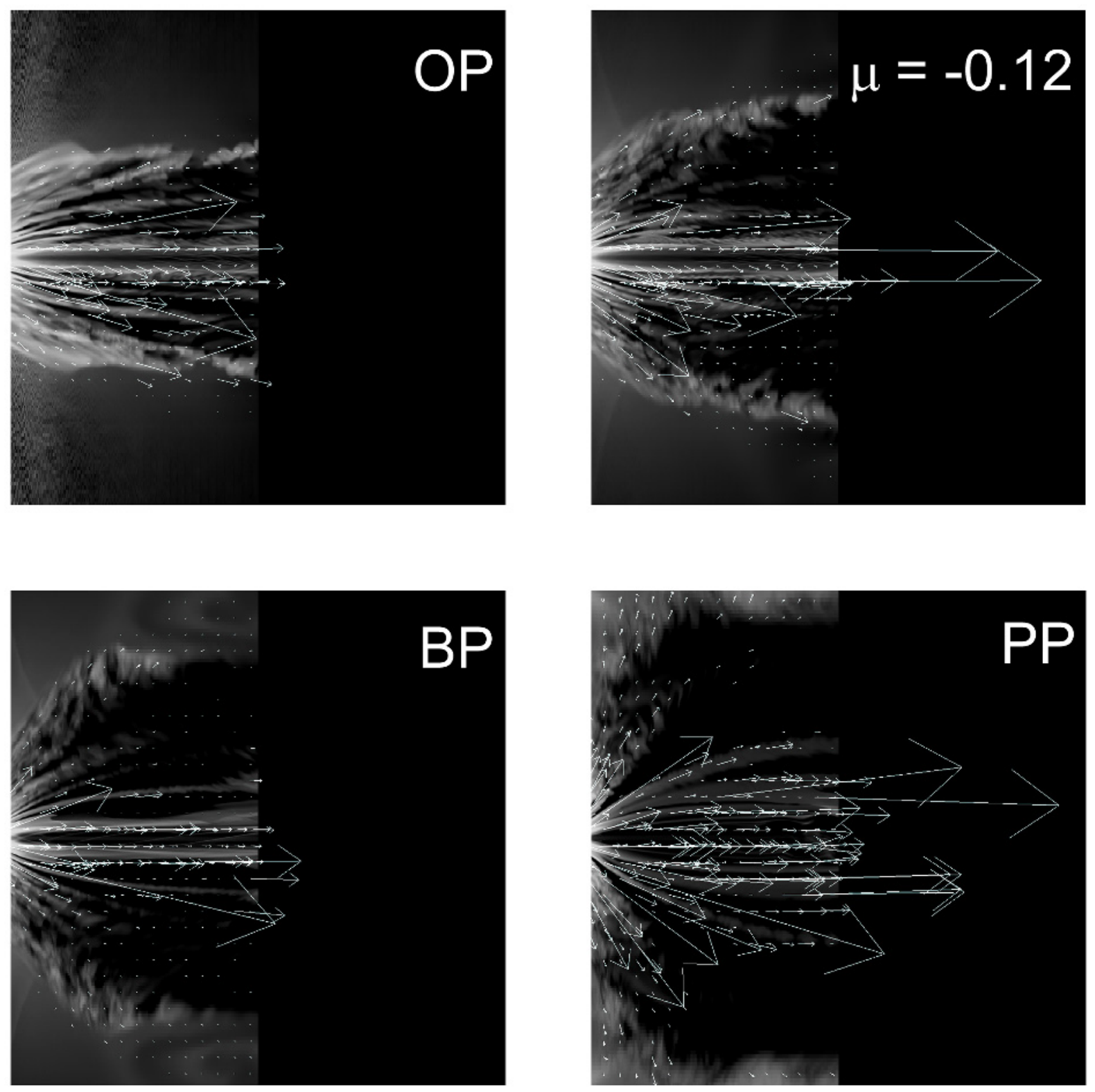

Figure 4. The momentum vectors overlaid on the density structure in a cut through the middle of the grid for the four simulations. The vectors are normalized for each figure individually, so the length of the vectors can not be compared between the different simulations.

is thus feasible according to our findings if one includes the Blandford-Znajek component.

\section{References}

[1] Blandford, R. D., Payne, D. R. 1982, MNRAS 199, 883

[2] Berger, E., Kulkarni, S. R., Pooley, G., et al. 2003, Nature, 426,154

[3] Blandford, R. D., \& Znajek, R. L. 1977, MNRAS, 179,433

[4] Chiaberge, M., Celotti, A., Capetti, A., \& Ghisellini, G. 2000, A\&A, 358, 104
[5] Jørgensen, M, Ouyed, R., \& Christensen, M, 2001, A\&A 379, 1170

[6] Kovalev, Y. Y., Lister, M. L., Homan, D. C., \& Kellermann, K. I. 2007, ApJL, 668, 27

[7] Misner, C. W., Thorne, K. S., \& Wheeler, J. A., 1973, Gravitation (San Francisco : W. H. Freeman and Co.)

[8] Ouyed, R., Pudritz, R. E., \& Stone, J. M. 1997, Nature, 385,409

[9] Ouyed, R. \& Pudritz, R. E. 1999, MNRAS, 309, 233

[10] Pelletier, G. \& Pudrtitz, R. E. 1992, ApJ, 394, 117

[11] Price, D. J., Pringle, J. E., \& King, A. R. 2003, MNRAS, 339, 1223 
[12] Staff, J. E., Niebergal, B. P., Ouyed, R., Pudritz, R. E., \& Cai, K. 2010, ApJ, 722, 1325 